\title{
Infinite-Dimensional Linear Dynamical Systems with Chaoticity *
}

\author{
Xin-Chu FU \\ Mathematics Institute \\ University of Warwick \\ Coventry CV4 7AL, UK \\ and \\ Wuhan Institute of Physics and Mathematics \\ The Chinese Academy of Sciences \\ P. O. Box 71010, Wuhan 430071, CHINA \\ Jinqiao DUAN \\ Department of Mathematical Sciences \\ Clemson University \\ Clemson, SC 29634, USA
}

22 December, 1997

\begin{abstract}
The authors present two results on infinite-dimensional linear dynamical systems with chaoticity. One is about the chaoticity of the backward shift map in the space of infinite sequences on a general Fréchet space. The other is about the chaoticity of a translation map in the space of real continuous functions. The chaos is shown in the senses of both Li-Yorke and Wiggins. Treating dimensions as freedoms, the two results imply that in the case of an infinite number of freedoms, a system may exhibit complexity even when the action is linear. Finally, the authors discuss physical applications of infinite-dimensional linear chaotic dynamical systems.
\end{abstract}

Key words: infinite-dimension, linearity, chaoticity

Running Head: Linear Chaotic Systems

\footnotetext{
${ }^{*}$ Research supported by the National Natural Science Foundation of China Grant No.19572075 for X. Fu, and by the National Science Foundation of USA Grant DMS-9704345 for J. Duan.
} 


\section{Introduction}

Since the mid-1970's, the experimental and theoretic research on chaotic phenomena and complexity of nonlinear systems has made great progress. A new science called nonlinear science has emerged. It is widely accepted that the nonlinearity is the source which leads a system to chaos. And in general, it is also thought that only nonlinear systems may be chaotic.

But what is the intrinsic connection between nonlinearity and chaoticity? Is there a linear system with chaoticity? We have discovered that the other factor, the dimension of the phase space of the system, plays an important role.

The relation among linearity, nonlinearity, dimensions and chaoticity may be roughly expressed as follows:

(A) Finite dimensions and linearity implies "nonchaoticity";

(B) Finite dimensions and chaoticity implies "nonlinearity";

(C) Chaoticity implies "finite dimensions and nonlinearity" or "infinite dimensions".

We thank the referees for pointing out relevant papers by Protopopescu, MacCluer, Gulisashvili, Chan and Shapiro (see [23], [3], [24], 222], and 14]). We constructed an infinitedimensional linear chaotic system in 1990, and earlier related results were presented during 1990-1993 ( see [8], [7], and [9] ). Godefroy and Shapiro ([13]) showed that a class of linear operators are chaotic. In particular, for the backward shift map $B$ in separable Hilbert spaces, the map $\lambda B$ with $|\lambda|>1$ is chaotic, as also discussed in Protopopescu [23] and MacCluer 22]. MacCluer [22 further showed that continuous semigroup $e^{t B}$, with $B$ the backward shift map in separable Hilbert spaces, is chaotic. He also showed that this is true for some other operators which are infinite series in terms of B.

Godefroy and Shapiro [13] showed that the translation operator in the Fréchet space of complex entire functions is chaotic, and this generalized Birkhoff's result [2]. Chan and Shapiro ([3]) further generalized this work to the space of slowly growing entire functions.

Linear chaotic infinite dimensional systems may be meaningful models for physical problems. For example, Protopopescu and Azmy [24] considered a infinite dimensional system of linear differential equations in the modeling of motions of particles of internal energy, and showed that it is chaotic. MacCluer 22 also pointed out that some linear models are useful for in modeling tool chatter, and modal interaction in structures. Gulisashvili and MacCluer [14] showed that a linear quantum harmonic oscillator is chaotic by using a result of Godefroy and Shapiro [13]

In all these cited papers, the chaos is in the sense of Wiggins 25 or Devaney [4] in terms of topological transitivity (dense orbits), sensitive dependence on initial conditions, and sometimes, dense periodic points.

In this paper, we will present two theorems on infinite-dimensional linear dynamical systems with chaoticity. In Section 2 we consider the backward shift map in the space of infinite sequences of elements from a general Fréchet space, and show that this backward shift map itself is chaotic in both senses of Wiggins, and of Li-Yorke 19 in terms of orbit separation of nonwandering non-periodic points. In Section 3 we show that the translation map in the space of real continuous functions is chaotic in both senses of Wiggins, and of Li-Yorke.

Note that we have only proved the backward shift itself is chaotic in the space of infinite sequences with elements from any Fréchet spaces, in both senses of Wiggins and of Li-Yorke. Godefroy and Shapiro's result (113]) implies that the backward shift multiplied by a constant exceed 1 is chaotic in the sense of Wiggins.

Our work on the translation map is posed in the space of real continuous functions. This space may be more natural for considering physical problems modeled by (linear) differential 
equations, than the space of complex entire functions as used by Godefroy and Shapiro [13]. Again, our work is in both senses of Wiggins and of Li-Yorke.

There are a few definitions of "chaos". Although the definition in Wiggins [25] seems a popular one, other definitions [19], [18], which capture or describe various different dynamical behavior of a system, are proposed and used in nonlinear science. Li-Yorke's definition of chaos does characterize the complexity of dynamical systems. For example, for onedimensional dynamical systems (the iteration of continuous maps on intervals), Li-Yorke's chaos is equivalent to having positive topological entropy. The same conclusion holds for subshifts of finite type. This motivates us to study chaos of the backward shift map and translation map in the sense of Li-Yorke. Our results provide tools to analyse chaotic behavior, especially Li-Yorke type, of infinite-dimensional linear physical systems. In Section 4, we will briefly discuss two linear physical systems which are chaotic in the senses of both Wiggins and Li-Yorke.

\section{Construction of a Linear Chaotic System: Discrete Case}

Before discussing infinite dimensional linear chaotic systems, we recall two definitions of chaos (see [19], [4], [25, 28]).

Definition 1. Let $(M, \rho)$ be a metric space, $F: M \rightarrow M$ be continuous. The discrete dynamical system $(M, F)$ is called chaotic in the sense of Li-Yorke if there exists an uncountable subset $S$ of nonwandering and non-periodic points such that the following conditions hold,

(i) $\limsup _{n \rightarrow+\infty} \rho\left(F^{n}(x), F^{n}(y)\right)>0$

(ii) $\liminf _{n \rightarrow+\infty} \rho\left(F^{n}(x), F^{n}(y)\right)=0 \quad, \quad \forall x, y \in S, x \neq y$.

Remark 1. Note that the Li-Yorke type chaos is characterized via orbit separation. The original characterization of chaos in Li-Yorke's theorem $([19])$ is via three conditions. The third one is :

$$
\text { (iii) } \limsup _{n \rightarrow+\infty} \rho\left(F^{n}(x), F^{n}(p)\right)>0,
$$

for $\forall x \in S$ and for any periodic point $p$. This condition means that there exist no asymptotically periodic points in $S$. From the conditions (i) and (ii) in Definition 1, it can be shown that $S$ contains at most one asymptotically periodic point. So the condition (iii) is not essential and then removable. This observation seems not well-known and we briefly prove it here (see [28] and 12] for detail). Namely, we show that, under conditions (i) and (ii), $S$ contains at most one asymptotically periodic point. Note that asymptotically periodic points are also nonwandering points. Suppose that $S$ contains two asymptotically periodic points $x_{1}, x_{2}$, i.e., there exist periodic points $p_{1}, p_{2}$ (which may or may not be the same) of $F$, such that

$$
\lim _{n \rightarrow+\infty} \rho\left(F^{n}\left(x_{i}\right), F^{n}\left(p_{i}\right)\right)=0, i=1,2 .
$$

Note that

$$
\begin{aligned}
& \rho\left(F^{n}\left(p_{1}\right), F^{n}\left(p_{2}\right)\right)-\rho\left(F^{n}\left(p_{1}\right), F^{n}\left(x_{1}\right)\right)-\rho\left(F^{n}\left(x_{2}\right), F^{n}\left(p_{2}\right)\right) \\
\leq & \rho\left(F^{n}\left(x_{1}\right), F^{n}\left(x_{2}\right)\right) \\
\leq & \rho\left(F^{n}\left(x_{1}\right), F^{n}\left(p_{1}\right)\right)+\rho\left(F^{n}\left(p_{1}\right), F^{n}\left(p_{2}\right)\right)+\rho\left(F^{n}\left(p_{2}\right), F^{n}\left(x_{2}\right)\right), \forall n \geq 0 .
\end{aligned}
$$


Hence, if $p_{1}=p_{2}$, then

$$
\lim _{n \rightarrow+\infty} \rho\left(F^{n}\left(x_{1}\right), F^{n}\left(x_{2}\right)\right)=0,
$$

which contradicts condition (i). If $p_{1} \neq p_{2}$, then

$$
\liminf _{n \rightarrow+\infty} \rho\left(F^{n}\left(x_{1}\right), F^{n}\left(x_{2}\right)\right)>0,
$$

which contradicts condition (ii). So $S$ can contain at most one asymptotically periodic point, and thus condition (iii) is not needed.

Definition 2. Let $M, \rho, F$ be the same as in Definition 1 , and $S \subseteq M$ be a compact invariant subset of $F$. $(M, F)$ is said to be chaotic in the sense of Wiggins, if

(a) $F$ has sensitive dependence on initial conditions on $S$; i,e., there exists $\delta>0$ such that for any $x$ in $S$ and any neighborhood $U$ of $x$, there exists $y \in U$ and $n \geq 0$ such that

$$
\rho\left(F^{n}(x), F^{n}(y)\right)>\delta
$$

(b) $F$ is topologically transitive on $S$; i.e., for any two open subsets $U$ and $V$ in $S$, there exists $k>0$, such that

$$
F^{k}(U) \cap V \neq \varnothing .
$$

Remark 2. For the case of a flow, it is not difficult to give corresponding definitions as Definition 1 and 2. In Definition 2, the case that $S$ is without interior points is a trivial case. We do not treat this special case.

In 跑, Devaney adds the third condition to Definition 2:

(c) The periodic points of $F$ are dense in $S$.

Remark 3. The conditions (b) and (c) imply condition (a) [1]. But the importance and relationship to chaos of condition (c) should not be neglected (see 25]).

Now we define a discrete infinite dimensional linear chaotic system.

Let $(X, d)$ be a Fréchet space over the complex field $C$ (i.e.,a complete linear metric space over $C$ ). We denote $\Sigma(X)$ the space of functions from the nonnegative integers to $X, c: \mathcal{Z}^{+} \rightarrow X$, where $c(i)$ is denoted by $c_{i}$ and $c$ may be denoted by $c=\left(c_{0}, c_{1}, \cdots\right)$, and define the backward shift $\sigma: \Sigma(X) \rightarrow \Sigma(X)$ by $(\sigma(c))_{i}=c_{i+1}$. And let $\Sigma(X)$ be endowed with the product topology with the metric

$$
\rho(x, y)=\sum_{i=0}^{+\infty} \frac{1}{2^{i}} \frac{d\left(x_{i}, y_{i}\right)}{1+d\left(x_{i}, y_{i}\right)}, \quad x=\left(x_{0}, x_{1}, \cdots\right), y=\left(y_{0}, y_{1}, \cdots\right) \in \Sigma(X) .
$$

This generalizes the usual symbolic dynamics system $\left.\left(\Sigma^{N}, \sigma\right)(\sqrt[25])\right]{)}$.

Remark 4. For a Fréchet space $(X, d)$, the metric $d$, or $d^{\prime}$ which is equivalent to $d$, is translation-invariant. So the metric $\rho$ on $\Sigma(X)$ may be regarded as translation-invariant.

Define the addition " $\oplus$ " and the scalar multiplication "." in $\Sigma(X)$ as follows:

$$
\begin{aligned}
& (x \oplus y)_{i}=x_{i}+y_{i} \\
& (\alpha \cdot x)_{i}=\alpha x_{i}
\end{aligned} \quad, \quad x, y \in \Sigma(X), \alpha \in C, i=0,1, \cdots,
$$

then it is not difficult to verify that $(\Sigma(X), \rho)$ is a Fréchet space over $C$. From

$$
\rho(\sigma(x), \sigma(y)) \leq 2 \rho(x, y),
$$


$\sigma$ is continuous. $(\Sigma(X), \sigma)$ is the general symbolic dynamics system (see [12] chapter 13 or [11]).

It may be verified that

$$
\sigma(\alpha \cdot x \oplus \beta \cdot y)=\alpha \cdot \sigma(x) \oplus \beta \cdot \sigma(y), \quad \forall x, y \in \Sigma(X), \forall \alpha, \beta \in C,
$$

that is, $\sigma: \Sigma(X) \rightarrow \Sigma(X)$ is a linear map, and $(\Sigma(X), \sigma)$ a linear dynamical system.

For the orbit $\left\{\sigma^{n}(x), n \geq 0\right\}$ from $x$ and the orbit $\left\{\sigma^{n}(y), n \geq 0\right\}$ from $y$, consider their linear combinations. We have

$$
\alpha \cdot \sigma^{n}(x) \oplus \beta \cdot \sigma^{n}(y)=\sigma^{n}(\alpha \cdot x \oplus \beta \cdot y),
$$

i.e., $\left\{\alpha \cdot \sigma^{n}(x) \oplus \beta \cdot \sigma^{n}(y), n \geq 0\right\}$ is the orbit from $\alpha \cdot x \oplus \beta \cdot y$. So, the system $(\Sigma(X), \sigma)$ satisfies the orbit superposition principle .

When $X$ is nontrivial, $\Sigma(X)$ is infinite-dimensional.

We denote $x=\left(x_{0}, x_{1}, \cdots\right)^{T} \in \Sigma(X)$ as an infinite-dimensional column vector. Define the infinite matrix

$$
A=\left(a_{i j}\right)_{i, j=0,1, \cdots,+\infty}
$$

where

$$
a_{i j}= \begin{cases}1, & j=i+1, \\ 0, & \text { otherwise. }\end{cases}
$$

Then the map $\sigma$ has a matrix representation

$$
\sigma(x)=A x,
$$

which can also be written as

$$
\sigma(x)=\left(\sum_{k=0}^{+\infty} a_{0 k} x_{k}, \sum_{k=0}^{+\infty} a_{1 k} x_{k}, \cdots\right) .
$$

For any $\lambda \in C, \lambda$ is an eigenvalue of $\sigma$, and there are infinitely many eigenvectors to an eigenvalue $\lambda$,

$$
x=\left(x_{0}, \lambda x_{0}, \lambda^{2} x_{0}, \cdots, \lambda^{k} x_{0}, \cdots\right), \quad \forall x_{0} \neq 0 .
$$

The following proposition is obvious:

Proposition 2.1. (1) $\forall \lambda \in C, \lambda$ is an eigenvalue of $\sigma$, and the corresponding eigenspace is isomorphic to $X$. (2) The spectrum of $\sigma$ consists of eigenvalues.

Many familiar operators have finite or infinite countable number of eigenvalues in discrete distribution. But the map $\sigma$ here has uncountable eigenvalues in continuum distribution. This is an indication that the system $(\Sigma(X), \sigma)$ may have complicated properties. The following two theorems justify this observation. The first result, Theorem 2.2, was included here to point out that the subshifts of $(\Sigma(X), \sigma)$ can be used as models of other continuous maps in infinite dimensional metric spaces.

Theorem 2.2(X.-C. Fu [8]). Let $(X, d)$ be a metric space. For any continuous map $\tau: X \rightarrow X$, there exists a subshift $\left(\Sigma_{\tau}, \sigma_{\tau}\right)$ of $(\Sigma(X), \sigma)$, such that $\tau$ is topologically conjugate to $\sigma_{\tau}$, where $\sigma_{\tau}$ is $\sigma$ restricted to $\Sigma_{\tau}$.

The above theorem shows the importance of subshifts of $(\Sigma(X), \sigma)$. And meanwhile it reveals the extraordinarily plentiful structure of subshifts in $(\Sigma(X), \sigma)$. Such a system $(\Sigma(X), \sigma)$, probably, should have chaotic properties. Indeed, we have:

Theorem 2.3. Suppose $(X, d)$ is a nontrivial separable Fréchet space; then the infinitedimensional linear dynamical system $(\Sigma(X), \sigma)$ is chaotic in the senses of both Li-Yorke and Wiggins. 
Proof. We remark that our result on the chaoticity of the backward shift is constructive and direct, and it appears that this result does not follow directly from Corollary 1.5 in Godefroy and Shapiro [13] as used in chaos proofs of [23], [3], [24, [22], and [14].

We first prove that $\sigma$ is chaotic in the sense of Li-Yorke, that is, we will construct an uncountable set satisfying the conditions in Definition 1.

Choose arbitrarily $a, b \in X, a \neq b$. And take an onto map

$$
\alpha:(0,1) \rightarrow X-\{a\} .
$$

$\forall r \in(0,1)$, define $x^{r}=\left(x_{0}^{r}, x_{1}^{r}, \cdots\right) \in \Sigma(X)$ as follows:

$$
\left\{\begin{array}{l}
x_{0}^{r}=b \\
x_{k^{2}}^{r}= \begin{cases}a, & \varphi(k, r)=1 \\
b, & \varphi(k, r)=0\end{cases} \\
x_{l}^{r}= \begin{cases}b, & k^{2}+1 \leq l \leq(k+1)^{2}-2 \\
\alpha(r), & l=(k+1)^{2}-1\end{cases}
\end{array}\right.
$$

where $k=1,2, \cdots, \varphi(k, r)=[k r]-[(k-1) r]$ (may be called the Zhou function (see 12 and [28])),[.] denotes the integral part of a real number. Then $x^{r}=\left(x_{0}^{r}, x_{1}^{r}, \cdots\right) \in \Sigma(X)$.

We denote $\Omega(\sigma)$ as the set of nonwandering points of $\sigma$ and $P(\sigma)$ the set of periodic points. Let $S=\left\{x^{r} \mid r \in(0,1)\right\}$; then $S \subseteq \Omega(\sigma)-P(\sigma)$ (Note that $\Omega(\sigma)=\Sigma(X)$ ).

Denote by $P\left(x^{r}, k\right)$ the number of $x_{l}^{r}$ 's which satisfy $x_{l}^{r}=a, 0 \leq l \leq k$. Then

$$
[\sqrt{k} r] \leq P\left(x^{r}, k\right) \leq[(\sqrt{k}+1) r]
$$

this implies

$$
\lim _{k \rightarrow+\infty} \frac{P\left(x^{r}, k\right)}{\sqrt{k}}=r .
$$

When $r_{1}, r_{2} \in(0,1)$ and $r_{1} \neq r_{2}$, we have $x^{r_{1}} \neq x^{r_{2}}$ from $(*)$. So $S$ is an uncountable subset in $\Sigma(X)$.

From the construction of $x^{r}$, only the entries of type $x_{k^{2}}^{r}$ may take the value $a$. Therefore, $\forall r_{1}, r_{2} \in(0,1), r_{1} \neq r_{2}$, there exist infinite number of positive integers $k_{n}, n=1,2, \cdots$, such that $x_{k_{n}^{2}}^{r_{1}} \neq x_{k_{n}^{2}}^{r_{2}}$, due to $(*)$. And the entries of the type $x_{k^{2}}^{r}$ only take the value $a$ or $b$, so

$$
\begin{gathered}
\limsup _{n \rightarrow+\infty} \rho\left(\sigma^{n}\left(x^{r_{1}}\right), \sigma^{n}\left(x^{r_{2}}\right)\right) \geq \limsup _{n \rightarrow+\infty} \rho\left(\sigma^{k_{n}^{2}}\left(x^{r_{1}}\right), \sigma^{k_{n}^{2}}\left(x^{r_{2}}\right)\right) \\
\geq \frac{d(a, b)}{1+d(a, b)}>0 .
\end{gathered}
$$

When $k^{2}+1 \leq l \leq(k+1)^{2}-2, x_{l}^{r_{1}}=x_{l}^{r_{2}}=b, \forall r_{1}, r_{2} \in(0,1)$. And $(k+1)^{2}-2-\left(k^{2}+1\right)=$ $2(k-1)$, so we have

$$
\begin{gathered}
\liminf _{n \rightarrow+\infty} \rho\left(\sigma^{n}\left(x^{r_{1}}\right), \sigma^{n}\left(x^{r_{2}}\right)\right) \leq \liminf _{k \rightarrow+\infty} \rho\left(\sigma^{k^{2}+1}\left(x^{r_{1}}\right), \sigma^{k^{2}+1}\left(x^{r_{2}}\right)\right) \\
\leq \liminf _{k \rightarrow+\infty} \frac{1}{2^{2(k-1)}}=0 .
\end{gathered}
$$

Therefore we have proven that

$$
\begin{gathered}
\limsup _{n \rightarrow+\infty} \rho\left(\sigma^{n}(x), \sigma^{n}(y)\right)>0, \quad \forall x, y \in S \text { with } x \neq y, \\
\liminf _{n \rightarrow+\infty} \rho\left(\sigma^{n}(x), \sigma^{n}(y)\right)=0, \quad \forall x, y \in S .
\end{gathered}
$$


That is, $(\Sigma(X), \sigma)$ is a chaotic system in the sense of Li-Yorke.

We now prove that $\sigma$ is chaotic in the sense of Wiggins, that is, we show that $\sigma$ has sensitive dependence and is topologically transitive on a compact invariant set.

Choose a compact subset $K$ of $X$ with more than one points, and define a subset $\Sigma(K)$ of $\Sigma(X)$ by:

$$
\Sigma(K)=\left\{x \in \Sigma(X) \mid x_{i} \in K, i=0,1, \cdots\right\},
$$

then $\Sigma(K)$ is a compact subset of $\Sigma(X)$ invariant under $\sigma$.

To continue the proof of Theorem 2.3, we need the following claim.

Claim: There exists a positive constant $\delta_{0}$ such that

$$
\forall a \in K, \exists b \in K, \text { we have } d(a, b)>\delta_{0} .
$$

Let us prove this claim. We first consider the case of $\inf _{a \in K} \sup _{b \in K} d(a, b)=+\infty$. Suppose in this case there is no $\delta_{0}$ satisfying $(* *)$. Thus, $\forall \eta>0, \exists a_{0} \in K, \forall b \in K, d\left(a_{0}, b\right) \leq \eta$. This leads to

$$
\inf _{a \in K} \sup _{b \in K} d(a, b) \leq \sup _{b \in K} d\left(a_{0}, b\right) \leq \eta<+\infty .
$$

which contradicts with $\inf _{a \in K} \sup _{b \in K} d(a, b)=+\infty$. Hence the claim is proved in this case.

Now we consider the case of $\inf _{a \in K} \sup _{b \in K} d(a, b) \neq+\infty$. we take $\delta_{0}=\frac{1}{2} \inf _{a \in K} \sup _{b \in K} d(a, b)$. Then there exist $a_{0}, b_{0} \in K$, such that $d\left(a_{0}, b_{0}\right)>0$. Thus $\forall a \in K$, we have

$$
\sup _{b \in K} d(a, b) \geq \max \left\{d\left(a, a_{0}\right), d\left(a, b_{0}\right)\right\} \geq \frac{d\left(a, a_{0}\right)+d\left(a, b_{0}\right)}{2} \geq \frac{1}{2} d\left(a_{0}, b_{0}\right)>0,
$$

and

$$
\inf _{a \in K} \sup _{b \in K} d(a, b) \geq \frac{1}{2} d\left(a_{0}, b_{0}\right)>0 .
$$

So $0<\delta_{0}<+\infty$. Therefore,

$$
\delta_{0}<\inf _{a \in K} \sup _{b \in K} d(a, b) \leq \sup _{b \in K} d(a, b), \forall a \in K .
$$

So $\forall a \in K, \exists b \in K$, such that $d(a, b)>\delta_{0}$. Therefore, the constant $\delta_{0}$ satisfying (**) really exists. This proves the claim $(* *)$.

We now continue the proof of Theorem 2.3. Let $\delta=\delta_{0} /\left(1+\delta_{0}\right)$; then for any $x \in \Sigma(K)$ and its any neighborhood $U$ in $\Sigma(K)$, take

$$
y^{(k)}=\left(x_{0}, x_{1} \cdots, x_{k}, y_{k+1}, \cdots\right),
$$

where $y_{k+1} \in K$ satisfies $d\left(x_{k+1}, y_{k+1}\right)>\delta_{0}$. When $k$ is big enough, we have $y^{(k)} \in U$ and

$$
\rho\left(\sigma^{k+1}(x), \sigma^{k+1}\left(y^{(k)}\right)\right)=\frac{d\left(x_{k+1}, y_{k+1}\right)}{1+d\left(x_{k+1}, y_{k+1}\right)}+\cdots>\frac{\delta_{0}}{1+\delta_{0}}=\delta .
$$

i.e., $\sigma$ has sensitive dependence on initial conditions on $\Sigma(K)$.

Because $X$ is separable and thus $K$ is separable as well, there exists a countable subset $A \subseteq K$, such that the closure of $A$ is $K$.

Denote $A=\left\{a_{0}, a_{1}, \cdots, a_{k}, \cdots\right\}$ and $A_{N}=\left\{a_{0}, a_{1}, \cdots, a_{N}\right\}, N \geq 1$. Let $M_{p q}^{N}$ be the $q$-th arrangement according to the lexicographic order of all repeatable words of length $p$ in the 
elements in $A_{N}, N \geq 1,1 \leq q \leq N^{p}, p=1,2, \cdots$. There are countable infinite elements $M_{p q}^{N}$. So we can arrange the elements $M_{p q}^{N}$ and denote them by new symbols: $B_{1}, B_{2}, \cdots, B_{k}, \cdots$. We form a symbol sequence $b$ by putting together all the words $B_{i}, i=1,2, \cdots$.

$\forall \varepsilon>0, \forall x=\left(x_{0}, x_{1}, \cdots, x_{k}, \cdots\right) \in \Sigma(K)$, for $x_{k} \in K$, there exist $y_{k} \in A$, such that

$$
d\left(x_{k}, y_{k}\right)<\frac{\varepsilon}{4}, \quad k=0,1, \cdots .
$$

Take $y=\left(y_{0}, y_{1}, \cdots, y_{k}, \cdots\right)$; then $\forall m>0$, by the construction of $b$, there is $k \geq 0$, such that the first $m+1$ entries of $y$ and $\sigma^{k}(b)$ agree, so

$$
\rho\left(y, \sigma^{k}(b)\right) \leq \frac{1}{2^{m}}
$$

i.e., the orbit $\left\{\sigma^{n}(b), n=0,1, \cdots\right\}$ can be arbitrarily close to $y$. So there exists $n \geq 0$, such that

$$
\rho\left(y, \sigma^{n}(b)\right)<\frac{\varepsilon}{2} .
$$

Therefore

$$
\rho\left(x, \sigma^{n}(b)\right) \leq \rho(x, y)+\rho\left(y, \sigma^{n}(b)\right)<\sum_{i=0}^{+\infty} \frac{1}{2^{i}} \frac{\varepsilon}{4+\varepsilon}+\frac{\varepsilon}{2}<\varepsilon .
$$

So, $\left\{\sigma^{n}(b), n=0,1, \cdots\right\}$ is a dense orbit in $\Sigma(K)$.

Hence, for any nonempty open subsets $U$ and $V$ in $\Sigma(K)$, there exist $n_{2}>n_{1}>0$, such that $\sigma^{n_{1}}(b) \in U$ and $\sigma^{n_{2}}(b) \in V$. Hence $\sigma^{n_{2}-n_{1}}(U) \cap V$ is not empty, and $\sigma$ is topologically transitive on $\Sigma(K)$.

Thus, $(\Sigma(X), \sigma)$ is a chaotic system in the sense of Wiggins.

The proof of Theorem 2.3 is complete.

\section{Construction of a Linear Chaotic System: Continuous Case}

Let $B=C^{0}(R)$, the space of real continuous functions on real axis $R$. With the metric

$$
\rho(f, g)=\sup _{a>0} \min \left\{\max _{|x| \leq a}|f(x)-g(x)|, \frac{1}{a}\right\},
$$

$B$ is a complete separable metric space. Moreover, $\rho$ is translation-invariant.

The addition and the scalar multiplication in $B$ are defined in usual fashion, i.e.,

$$
\begin{aligned}
& (f \oplus g)(x)=f(x)+g(x) \\
& (\alpha \cdot f)(x)=\alpha f(x)
\end{aligned}, \quad \forall f, g \in B, \alpha, x \in R
$$

then $B$ is an infinite-dimensional separable Fréchet space.

Define a map $b: B \times R \rightarrow B$ as follows:

$$
\begin{aligned}
& b(f, t)=f_{t} \\
& f_{t}(x)=f(x+t)
\end{aligned}, \quad \forall t, x \in R,
$$

then it may be verified that $b: B \times R \rightarrow B$ is a flow in the infinite-dimensional phase space $B$. This dynamical system, denoted by $B_{t}$, is called the Biebutov system [27]. 
It may be verified that

$$
b(\alpha \cdot f \oplus \beta \cdot g, t)=\alpha \cdot b(f, t) \oplus \beta \cdot b(g, t), \quad \forall f, g \in B, \forall \alpha, \beta, t \in R,
$$

so $B_{t}$ is a linear system. And the above equality indicates that the linear combination $\alpha \cdot b(f, t) \oplus \beta \cdot b(g, t)$ of the orbits from $f$ and $g$ in $B$ is exactly the orbit from $\alpha \cdot f \oplus \beta \cdot g$. This means the system $B_{t}$ satisfies the solution superposition principle.

The system $B_{t}$ may be treated as a continuous shift system. Many topological dynamical systems may be embedded into $B_{t}$.

Consider the discrete case, i.e., the restriction of $f \in C^{0}(R)$ on $\{0, \pm \tau, \pm 2 \tau, \cdots\}$, where $\tau>0$. Then $f$ corresponds to a bi-infinite sequence $f^{\tau}$ :

$$
f^{\tau}=(\cdots, f(-\tau), f(0) ; f(\tau), \cdots) .
$$

Let the time $t$ run discrete values $n \tau, n=0, \pm 1, \pm 2, \cdots$, then

$$
b\left(f^{\tau}, n \tau\right)=f_{n}^{\tau},
$$

where

$$
f_{n}^{\tau}=(\cdots, f((n-1) \tau), f(n \tau) ; f((n+1) \tau), \cdots) .
$$

Denote $\sigma=b(\cdot, \tau)$, and

$$
\Sigma\left(C^{0}(R)\right)=\left\{f_{n}^{\tau} \mid n \geq 0, f \in C^{0}(R)\right\},
$$

then $\left(\Sigma\left(C^{0}(R)\right), \sigma\right)$ is a two-sided symbolic dynamics system.

A subsystem $M_{t}$ of $B_{t}$ is constructed in [27], where $M_{t}$ is complete and all orbits in $M_{t}$ are asymptotic.

So the system $B_{t}$ possesses very complex structure. The further analysis shows that $B_{t}$ has chaotic properties, i.e., we have:

Theorem 3.1. The infinite-dimensional linear dynamical system $B_{t}$ is chaotic in the senses of both Li-Yorke and Wiggins.

Proof. We first prove that $B_{t}$ is chaotic in the sense of Li-Yorke.

Construct a subset $S$ in $B$ as follows:

$$
\begin{gathered}
S=\left\{f_{[r]} \mid r \in(0,1)\right\} \\
f_{[r]}(x)= \begin{cases}0, \quad x \in\left[-\left(a+\frac{1}{2}\right),-\left(a-\frac{1}{2}\right)\right] \cup\left[a-\frac{1}{2}, a+\frac{1}{2}\right] \\
1, \quad x= \pm(a \pm 1) & x \in\left(-(a+1),-\left(a+\frac{1}{2}\right)\right) \\
\text { continuous interpolation, } & \cup\left(-\left(a-\frac{1}{2}\right),-(a-1)\right) \\
& \cup\left(a-1, a-\frac{1}{2}\right) \cup\left(a+\frac{1}{2}, a+1\right) \\
1, \text { all other cases } & \end{cases}
\end{gathered}
$$

where $a=k^{2}, k^{\prime} s$ are positive integers satisfying $\varphi(k, r)=1$, and $\varphi(k, r)=[k r]-[(k-1) r]$ is the Zhou function [28].

Denote by $P\left(f_{[r]}, A\right)$ the number of intervals contained in $\left[-\left(A+\frac{1}{2}\right), A+\frac{1}{2}\right]$ in which $f_{[r]}(x) \equiv 0$, where $A$ is a positive integer. Then

$$
\begin{gathered}
{[\sqrt{A} r] \leq P\left(f_{[r]}, A\right) \leq[(\sqrt{A}+1) r],} \\
\lim _{A \rightarrow+\infty} \frac{P\left(f_{[r]}, A\right)}{2 \sqrt{A}}=r .
\end{gathered}
$$


So $f_{\left[r_{1}\right]} \neq f_{\left[r_{2}\right]}$ for $r_{1} \neq r_{2}$, and $S$ is uncountable. Furthermore, when $r_{1} \neq r_{2}$, there exist an infinite number of $t_{n}, n=1,2, \cdots, t_{n} \rightarrow \infty(n \rightarrow+\infty)$, such that $f_{\left[r_{1}\right]}\left(t_{n}\right) \neq f_{\left[r_{2}\right]}\left(t_{n}\right)$ (Otherwise, we have $P\left(f_{\left[r_{1}\right]}, A\right)=P\left(f_{\left[r_{2}\right]}, A\right)+C$, this implies $r_{1}=r_{2}$, a contradiction). So

$$
\limsup _{t \rightarrow \infty} \rho(b(f, t), b(g, t)) \geq 1>0, \quad \forall f, g \in S, f \neq g .
$$

When $x \in\left[k^{2}+1,(k+1)^{2}-1\right] \cup\left[1-(k+1)^{2},-k^{2}-1\right], f_{[r]}(x)=1, \forall r \in(0,1)$. And the lengths of the intervals $\left[k^{2}+1,(k+1)^{2}-1\right]$ and $\left[1-(k+1)^{2},-k^{2}-1\right]$ are $2 k-1 \rightarrow$ $+\infty(k \rightarrow+\infty)$. Thus

$$
\liminf _{t \rightarrow \infty} \rho(b(f, t), b(g, t))=0, \quad \forall f, g \in S .
$$

$\forall f \in B, a>0$, take $f^{[a]} \in B$ as follows:

$$
f^{[a]}(x)=\left\{\begin{array}{l}
f(x), \quad x \in[-a, a] \\
f(x-3 a), \quad x \in[2 a, 4 a] \\
\text { continuous extension, } \quad x \in(-\infty,-a) \cup(a, 2 a) \cup(4 a,+\infty)
\end{array}\right.
$$

then

$$
\begin{gathered}
\rho\left(f, f^{[a]}\right) \leq \frac{1}{a}, \\
\rho\left(f, b\left(f^{[a]}, 3 a\right)\right) \leq \frac{1}{a} .
\end{gathered}
$$

In the following, $\Omega\left(B_{t}\right)$ is the set of nonwandering points of $B_{t}$, while $P\left(B_{t}\right)$ is the set of periodic points. From $\Omega\left(B_{t}\right)=\{f \in B \mid \forall U \in o(f), \exists g \in U, t>0$, such that $b(g, t) \in U\}$, we have $\Omega\left(B_{t}\right)=B$. Where $o(f)$ denotes the collection of neighbourhoods of $f$. Obviously, $S \cap P\left(B_{t}\right)=\varnothing$. So $S \subset \Omega\left(B_{t}\right)-P\left(B_{t}\right)$.

Therefore, $B_{t}$ is chaotic in the sense of Li-Yorke.

We now prove that $B_{t}$ is chaotic in the sense of Wiggins.

$\forall f \in B, \forall a>1$, let $f^{(a)} \in B$ as follows:

$$
f^{(a)}(x)=\left\{\begin{array}{l}
f(x), x \in[-a, a] \\
f(x)+1, x \in[a+1, a+3] \\
\text { continuous extension, } x \in(-\infty,-a) \cup(a, a+1) \cup(a+3,+\infty)
\end{array}\right.
$$

then $f^{(a)}$ may enter an arbitrarily small neighbourhood of $f$ for $a$ big enough. However,

$$
\rho\left(b(f, a+2), b\left(f^{(a)}, a+2\right)\right)=1 .
$$

This implies that the system $B_{t}$ has sensitive dependence on initial conditions.

From the separability of $B$, there exists a countable subset $F=\left\{f_{(k)} \mid k=0,1, \cdots\right\} \subset B$, such that $F$ is dense in $B$.

For a positive integer $n$, denote by $\varphi_{k}^{n}(x)$ the continuous function on the interval $[-(n+$ 1), $n+1]$ :

$$
\varphi_{k}^{n}(x)=\left\{\begin{array}{l}
f_{(k)}(x), \quad x \in[-n, n] \\
0, \quad x= \pm(n+1) \\
\text { continuous interpolation, } \quad x \in(-(n+1),-n) \cup(n, n+1)
\end{array}\right.
$$

then $\left\{\varphi_{k}^{n} \mid n=1,2, \cdots ; k=0,1, \cdots\right\}$ is countable; and

$$
\left\{\left\{\varphi_{k}^{n}(x) \mid x \in[-(n+1), n+1]\right\} \mid n=1,2 \cdots ; k=0,1, \cdots\right\}
$$


is a countable collection of curve segments. Make suitable translation transformation to the intervals of definition of the curve segments, such that the new intervals of definition cover the real number axis $R$ without overlaps. Correspondingly, the new curve segments join together and form a continuous curve on $R$, denoted by $\{\varphi(x) \mid x \in R\}$. Then the orbit $\{b(\varphi, t) \mid t \in R\}$ from $\varphi$ is dense in $B$.

For $D>0$, we take $K=\{f \in B|| f(x) \mid \leq D, \forall x \in R\}$, then $K$ is a compact invariant subset of the flow $b: B \times R \rightarrow B$. From the above discussion, the restricted flow $b_{1}$ : $K \times R \rightarrow K$ has sensitive dependence on initial conditions and is topologically transitive.

So $B_{t}$ is a chaotic system in the sense of Wiggins.

This completes the proof of Theorem 3.1.

\section{Discussions and Applications}

As we know, finite-dimensional linear systems are nonchaotic. Dimensions may be treated as freedoms. The existence of infinite-dimensional linear chaotic systems indicates that if there are infinite number of freedoms, then a system may possibly create complexity even if the map (or flow) is linear. The discussions in this paper suggest that the deeper understanding on the structure of infinite-dimensional linear topological spaces and the properties of continuous linear maps on the spaces should also be an important research subject in nonlinear science. In [10] some new concepts and results are presented, and some intrinsic connections among dimensions of phase space, linearity, nonlinearity and complexity of a system are explored.

On the other hand, not every infinite-dimensional linear dynamical system is chaotic. For example, the global attractor of the infinite-dimensional linear dynamical system $\left(l^{2}, \sigma\right)$ discussed in 10 is a one-point set $\{\theta\}$. So the chaoticity of an infinite-dimensional linear dynamical system relates to the structure of phase space of the system. Moreover, a large class of quantum mechanical systems are not chaotic (see, [17).

Gulisashvili and MacCluer (14) showed that the annihilation operator (a weighted shift operator) for a linear quantum harmonic oscillator is chaotic in the sense of Wiggins (or

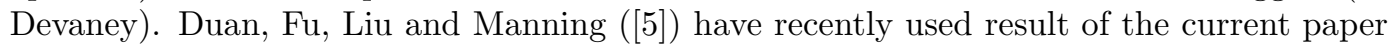
to prove that this annihilation operator is also chaotic in the sense of Li-Yorke. An unforced quantum harmonic oscillator, i.e., a very small frictionless mass-spring system, is modeled by the Schrödinger equation ([20])

$$
i \hbar \psi_{t}=-\frac{\hbar^{2}}{2 m} \psi_{x x}+\frac{k}{2} x^{2} \psi
$$

with wave function $\psi(x, t)$, displacement $x$, mass $m$, stiffness $k$ and Planck number $\hbar$. The nondimensionalized stationary states in the separable Hilbert space $X=L^{2}(-\infty, \infty)$ form an orthonormal basis

$$
\psi_{n}(x)=e^{-x^{2} / 2} H_{n}(x) / \sqrt{\sqrt{\pi} 2^{n} n !}, n=0,1, \cdots,
$$

where

$$
H_{n}(x)=(-1)^{n} e^{x^{2}} \frac{d^{n}}{d x^{n}} e^{-x^{2}},
$$

is the $n$th Hermite polynomial. The natural space for the quantum harmonic oscillator is the Schwartz class $F$ of rapidly decreasing functions in $X=L^{2}(-\infty, \infty)$ as defined later. Gulisashvili and MacCluer (14) defined a linear, closed, unbounded, weighted backward shift $B$ on $\mathrm{F}$ by

$$
B: F \rightarrow F,
$$




$$
B \psi_{n} \equiv \frac{1}{\sqrt{2}}\left(x+\frac{d}{d x}\right) \psi_{n}=\sqrt{n} \psi_{n-1} .
$$

$B$ has no resolvent set since every complex number $\lambda$ is in the point spectrum of $B$. By using a result of Godefroy and Shapiro ([13]), Gulisashvili and MacCluer ([14]) have shown that the shift operator $B$ is chaotic (顿), namely, it has topological transitivity (dense orbits), sensitivity to initial conditions (orbit divergence), and density of periodic points.

In terms of the orthonormal basis $\left\{\psi_{n}\right\}$, the Schwartz class $F$ can be written as $(14)$

$$
F=\left\{\phi \in L^{2}(-\infty, \infty): \phi=\sum_{n=0}^{\infty} c_{n} \psi_{n}, \sum_{n=0}^{\infty}\left|c_{n}\right|^{2}(n+1)^{r}<\infty, \forall r \geq 0\right\} .
$$

$F$ is an infinite-dimensional Fréchet space with topology defined by the system of semi-norms $p_{r}(\cdot)$ of the form $([26])$

$$
p_{r}(\phi)=p_{r}\left(\sum_{n=0}^{\infty} c_{n} \psi_{n}\right)=\left(\sum_{n=0}^{\infty}\left|c_{n}\right|^{2}(n+1)^{r}\right)^{1 / 2}, \quad r \geq 0 .
$$

This topology on $F$ is also given by the metric $\rho$

$$
\rho(\phi, \psi)=\sum_{m=0}^{\infty} 2^{-m} p_{m}(\phi-\psi) \cdot\left(1+p_{m}(\phi-\psi)\right)^{-1} .
$$

Fix $\theta \in(0,1)$ and define $\phi^{\theta}=\sum_{n=0}^{\infty} c_{n}^{\theta} \psi_{n}$ by

$$
\left\{\begin{array}{l}
c_{0}^{\theta}=0 \\
c_{n}^{\theta}=\left\{\begin{array}{cl}
1 / \sqrt{n !} & \text { if } n=k^{2},[k \theta]-[(k-1) \theta]=1 \\
0 & \text { otherwise },
\end{array}\right.
\end{array}\right.
$$

where $k=1,2, \cdots$, and [.] denotes the integer part of a real number.

Let $S=\left\{\phi^{\theta}: \theta \in(0,1)\right\}$. By using the idea and result in Section 2, it is shown (阿) that all points in $S$ are nonwandering and nonperiodic, and $S$ is a chaotic set for $B$, i.e., the weighted shift operator $B$ is chaotic in the sense of Li-Yorke.

Protopopescu (23) considered an infinite-dimensional linear rate equation arising in the modeling of particle distribution in statistical mechanics, and showed that it is chaotic in the sense of Wiggins. Liu, Fu and Duan [21] have recently used idea and result in Section 3 to show that this linear rate equation is also chaotic in the sense of Li-Yorke.

Duan, Fu and Lawson [6] also discussed the chaotic trajectory in the quantum state space of an arbitrary solution to the Schrödinger equation for Hydrogen atom under the repeated application of ladder operators, which shift the eigenfunctions $u_{n}$ of the Schrödinger equation into $u_{n-1}$ or $u_{n+1}$.

It would be interesting to see other linear physical models which are chaotic in the sense of Li-Yorke or Wiggins.

\section{Acknowledgments}

The authors would like to express their thanks to Prof. Zhang Zhi-Fen at Beijing University for her useful materials on the Biebutov system. They were grateful to the referees' helpful comments and useful suggestions as well as pointing out papers or books by Protopopescu, Azmy, MacCluer, Gulisashvili, Chan and Shapiro, and Ingraham. 


\section{References}

[1] J. Banks, J. Brooks, G. Cairns, G. Davis and P. Stacey, On Devaney's definition of chaos, Amer. Math. Monthly, 99(1992),332-334.

[2] G. D. Birkhoff, Démonstration d'un théoreme elementaire sur les fonctions entiéres, $C$. R. Acad. Sci. Paris 189 (1929), 473-475.

[3] K. C. Chan and J. H. Shapiro, The cyclic behavior of translation operators on Hilbert spaces of entire functions, Indiana Univ. Math. J., 40 (1991), 1421-1449.

[4] R. L. Devaney, An Introduction to Chaotic Dynamical Systems, Addison-Wesley, 1989.

[5] J. Duan, X.-C. Fu, P.-D. Liu, and A. K. Manning, On a linear chaotic quantum harmonic oscillator, submitted, 1997.

[6] J. Duan, X.-C. Fu and J. Lawson, Chaotic operators from quantum mechanics, preprint, 1997.

[7] X.-C. Fu, The general symbolic dynamics and its applications I, Invited talk, The National Conference on Nonlinear Dynamics, University of Science and Technology of China, May 1990.

[8] X.-C. Fu, The general symbolic dynamics and its applications II, in Nonlinear Oscillations, Bifurcations and Chaos (Chinese), Tianjin University Press, China, 1992.

[9] X.-C. Fu, Linearity, nonlinearity and chaoticity, Invited talk, The National Conference on Nonlinear Science, Shanghai, July. 1991. Also see Research on New Subjects, China Science and Technology Press, Beijing,1993.

[10] X.-C. Fu, The complexity of a class of shift systems, in Selected Scientific Research Papers (Chinese), China Science and Technology Press, Beijing, 1994.

[11] X.-C. Fu and H.-W. Chou, Chaotic behaviour of the general symbolic dynamics, Appl. Math. and Mech. 13(1992), 117-123.

[12] X.-C. Fu, H.-W. Chou, and K.-H. Xu, Bifurcations, Chaos, and Symbolic Dynamics (Chinese), Wuhan University Press, China, 1993.

[13] G. Godefroy and J. H. Shapiro, Operators with dense, invariant cyclic vector manifolds, J. Funct. Anal., 98(1991),229-269.

[14] A. Gulisashvili and C. R. MacCluer, Linear chaos in the unforced quantum harmonic oscillator, J. Dyn. Systems, Measurement and Control, 118 (1996), 337-338.

[15] D. A. Herrero, Limits of hypercyclic and supercyclic operators, J. Funct. Anal., 99 (1991), 179-190.

[16] D. A. Herrero, Triangular operators, Bull. London Math. Soc., 23(1991),513-554.

[17] R. L. Ingraham, A Survey of Nonlinear Dynamics, World Scientific, Singapore, 1992.

[18] U. Kirchgraber and D. Stoffer, On the definition of chaos, Z. Angew. Math. Mech., 69 (1989), 175-185.

[19] T.-Y. Li and J. A. Yorke, Period three implies chaos, Amer. Math. Monthly, 82(1975), 985-992 
[20] R. L. Liboff, Introductory Quantum Mechanics, 2nd ed., Addison-Wesley, 1992.

[21] Z.-R. Liu, X.-C. Fu, and J. Duan, Chaotic linear rate equations in Banach spaces, submitted to Advances in Differential Equations, 1997.

[22] C. R. MacCluer, Chaos in linear distributed systems, J. Dyn. Systems, Measurement and Control, 114 (1992), 322-324.

[23] V. Protopopescu, Linear vs. nonlinear and infinite vs. finite: An interpretation of chaos, Oak Ridge National Laboratory Report, TM-11667, Oak Ridge, Tennessee, USA, 1990.

[24] V. Protopopescu and Y. Y. Azmy, Topological chaos for a class of linear models, Math. Models and Methods in Appl. Sci. 2(1992), 79-90.

[25] S. Wiggins, Introduction to Applied Nonlinear Dynamical Systems and Chaos, SpringerVerlag, New York, 1990.

[26] K. Yosida, Functional Analysis, 4th Edition, Springer-Verlag, 1978.

[27] Z.-F. Zhang, On an example of asymptotic orbits, in Selected Papers on Differential Equations (Chinese), China Science Press, 1963.

[28] Z.-L. Zhou, Chaos and totally chaos, Science Bulletin (China), 32(1987), 248-250. 